\title{
Angst kann uns einander näherbringen
}

\section{Gregor Hasler}

Prof. Dr. med., Chefarzt Universitätsklinik für Psychiatrie Bern, ausserordentlicher Professor und Leiter der Abteilung für Molekulare Psychiatrie, Universität Bern, Präsident der Schweizer Gesellschaft für Bipolare Störungen

\begin{abstract}
Angst kann über kulturelle Grenzen hinweg verschiedene Ursachen haben - als Grundgefühl der Verunsicherung ist sie jedoch universell. Eine Tatsache, die Fachleute zur Verbesserung ihres Gesundheitswesens in Bezug auf Psychiatrie, Psychotherapie und Psychosomatik nutzen sollten - nicht nur im Iran.
\end{abstract}

\begin{abstract}
Was sind die Ursachen von Angststörungen? Warum nehmen sie zu? Mein Vortrag auf dem «Second International Anxiety Congress» in Teheran handelt - wie alle hier - von der Angst. Als ängstliches Kind musste ich mich wohl oder übel schon früh mit dem Grundgefühl der Verunsicherung auseinandersetzen. Viele Menschen schämen sich wegen ihrer Ängste und verheimlichen ihre Probleme selbst in einer vertrauensvollen Arzt-Patient-Beziehung.
\end{abstract}

Iraner wollen Psychiatrie und Psychosomatik mehr Beachtung schenken.

Trotz jahrelanger Beschäftigung mit der Angst und angstlösender Therapien war ich nun unangenehm und körperlich beunruhigt, als ich zu der Veranstaltung geflogen bin. Wenige Tage zuvor hatten islamische Terroristen einen Briten vor laufender Kamera geköpft. Will ich jetzt wirklich in Teheran sein? Ja, ich habe mich dafür entschieden. Mich freut nicht nur, dass man im Iran meine Arbeiten über die Neu-

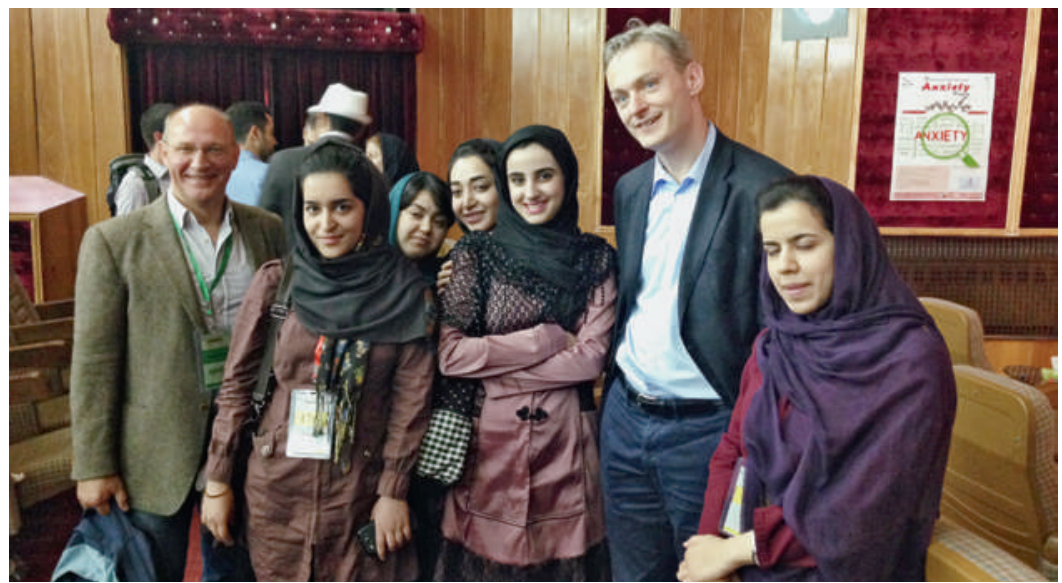

Iranische Psychologie-Studentinnen, der Basler Psychologe Serge Brand (links) und Gregor Hasler am zweiten Internationalen Angst-Kongress in Teheran.

\section{Summary}

Congrès de psychiatrie en Iran: la peur peut nous rapprocher

Quelles sont les raisons de la peur et des troubles psychosomatiques en Iran? Que nous apportent les échanges interculturels en la matière? Le compte rendu de Gregor Hasler du deuxième congrès international sur la peur qui s'est tenu à Téhéran soulève ces questions. La guerre entre I'Iran et I'Irak, le tremblement de terre à Bam, la fréquence des accidents de la circulation, l'insécurité économique et politique, et la fin des réseaux et des rôles traditionnels dans la société contribuent de manière significative à la hausse des troubles de la peur en Iran. Les Iraniens réagissent à ces défis en étoffant leur système de santé en matière de psychiatrie, de psychothérapie et de psychosomatique. Les échanges interculturels entre Américains, Européens et Iraniens montrent de manière frappante que la peur est universelle. Ses causes peuvent être différentes, mais le sentiment éprouvé reste le même. Il est capital que le dialogue international aborde les sujets qui nous rapprochent, et nous, médecins, pouvons apporter une pierre à cet édifice.

robiologie der Angst schätzt. Es ist eine grossartige Idee, sich als Europäer gemeinsam mit amerikanischen und iranischen Kollegen über Ängste auszutauschen, weil kulturelle Faktoren bei der Entstehung von Angststörungen eine immer grössere Rolle spielen.

Ein Sprecher des iranischen Parlaments, der den ersten Vortrag des Kongresses hält, kennt ein einfaches Mittel gegen Angst: Religion. Im Glauben an Allah würden sich unsere Ängste auflösen. Er betont die grosse Bedeutung von Familie und Gemeinschaft. Die Angst sei ein Resultat unserer Ungläubigkeit und der 
westlichen Kultur. Er spricht schnell und laut, der Übersetzer verliert den Faden. Am Schluss lobt der Mann das Engagement von Ärzten und Psychologen. Wie er die Brücke von der Religion zur Psychiatrie geschlagen habe, frage ich die ältere Dame neben mir. Die Pionierin der Familientherapie in Teheran gibt zu, nicht zugehört zu haben. Es spiele auch keine Rolle. Die Bedeutung der Psychotherapie nehme im Iran stark zu, der Kongress werde vom Staat finanziert.

\section{Religion ist kein Garant für psychische Stabilität}

Wie alle Frauen hier ist sie sorgfältig verschleiert. Auch eine US-Kollegin, Expertin für Zwänge und Perfektionismus, muss Schleier tragen. Sie sagt, die Kopfbedeckung störe, je länger, desto mehr, und zwar nicht nur mental, sondern auch physisch. Sie möge es, ihren Kopf im Freien zu haben. Eine Iranerin erklärt, dass man die Mädchen sehr früh an die Kopfbedeckung gewöhnen müsse, am besten ab dem fünften Lebensjahr. Der Schleier habe aber nicht nur Nachteile. Er reduziere die Rivalität unter Frauen. Eine britische Studie habe zudem gezeigt, dass verschleierte Frauen ein positiveres Körpergefühl und weniger Angst hätten, äusserlich nicht zu genügen.

Ein erfahrener Forscher berichtet von seiner Studie zu Ängsten in Iran. Junge Leute hätten vor allem Zukunftsängste, weil die Arbeitssituation prekär sei. Ältere hätten eher Existenzängste, weil sich die traditionellen sozialen Netze allmählich auflösten und die Altersabsicherung durch Institutionen gering sei. Ferner sei die Rechtssicherheit gefährdet, die Gesetze seien immer weniger verbindlich, die Religion werde von der Führungsschicht laufend neu interpretiert. Dies führe zu einer allgemeinen Desorientierung. Die religiöse Erziehung führe nicht zu mehr Sicherheit, im Gegenteil. Die Religion sei immer weniger eine

Psychotherapie hilft, kulturell bedingte Konflikte zwischen Tradition und Selbstverwirklichung zu lösen.

Quelle innerer Werte und innerer Unabhängigkeit, sondern bloss etwas Äusserliches, eine leere Hülle. Dies untergrabe die psychische Widerstandskraft.

Konflikte um die Rolle der Frau seien ein wichtiges Thema in Therapien, sagt eine iranische Psychotherapeutin. Viele Frauen seien gut ausgebildet, aufgeschlossen, freiheitsliebend und liberal. Zugleich seien sie in ihrer traditionellen Rolle eine wichtige Stütze der Gesellschaft.

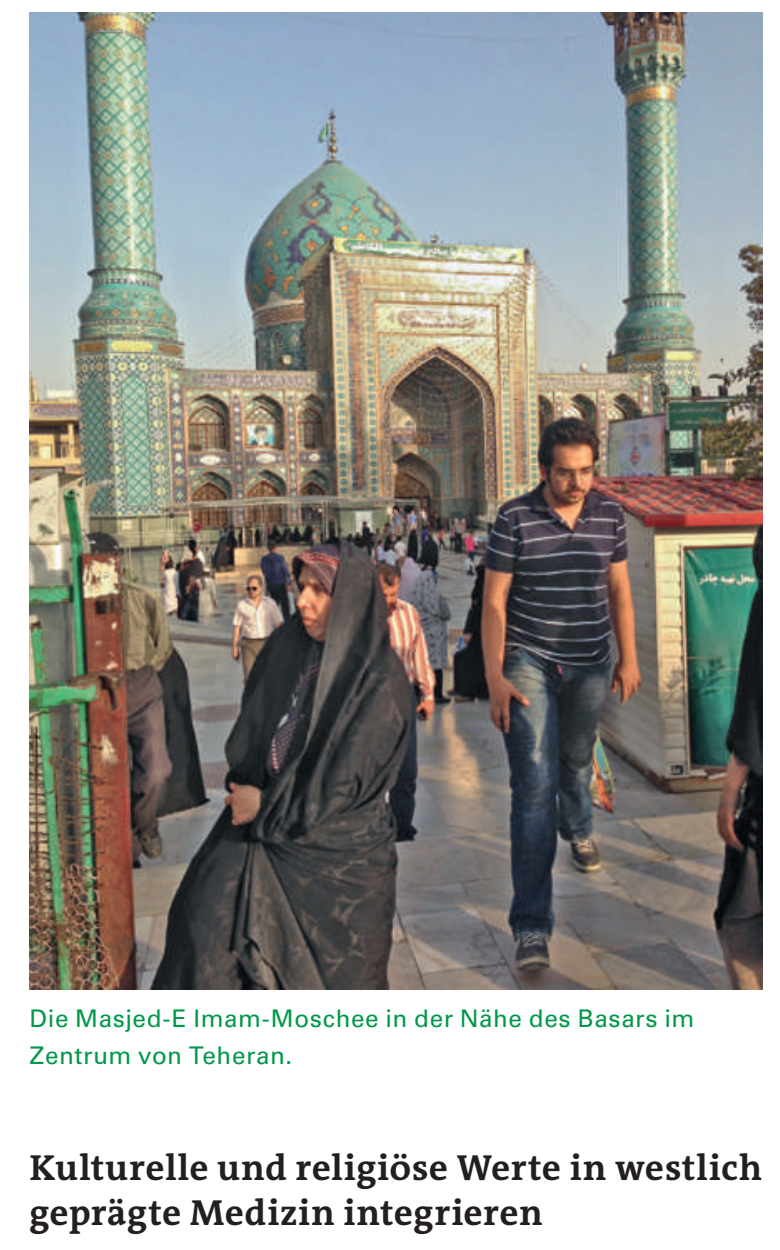

Bedeutsam sei dies vor allem in Familien, die vom Land in die Stadt ziehen. Das Aufeinanderprallen ländlicher Traditionen mit der zunehmend westlich geprägten städtischen Kultur überfordert viele: Sie entwickeln Angststörungen, oder ihre Verunsicherung führe zu psychosomatischen Beschwerden.

Im Treppenhaus spricht mich eine Psychotherapeutin in fliessendem Deutsch an. Sie teile meine Meinung, man solle weniger Psychopharmaka verschreiben. Mit Medikamenten und starren religiösen Vorstellungen werde man den Psychiatrie-Patienten im Iran nicht gerecht. Die Vorstellung, dass Islam und Individualismus nicht zusammengehen, hält sie für ein Vorurteil des Westens. Der Ausbau der Psychotherapie im Land sei entscheidend, um Konflikte zwischen Tradition und Selbstverwirklichung zu lösen. Aber auch die Ärztinnen und Ärzte müssten einen Beitrag dazu leisten, indem sie sich mehr Zeit nehmen würden, um auf die komplexen psychosozialen Probleme ihrer Patienten einzugehen. Sie schildert, wie Gespräche mit der Grossfamilie oft eine wichtige Wendung im Krankheitsverlauf bringen: Die verständnisvolle Meinung einer Grossmutter könne ausschlaggebend sein. Im Westen hat die Grossfamilie an Bedeutung stark abgenommen. Es kommt nur selten 
vor, dass ein Patient den Wunsch hat, sein psychiatrisches oder medizinisches Problem gemeinsam mit seiner Grossfamilie zu besprechen.

Generationenkonflikte kämen sehr häufig vor, junge Iraner seien zunehmend hedonistisch eingestellt. Die Religion versuche der Ausrichtung der jungen Generation an westlichen Werten entgegenzuwirken, indem sie sich über die Politik zunehmend in persönliche Dinge einmische. Familiäre Konflikte würden dadurch gesamtgesellschaftliches Ausmass annehmen. Dies führe zu enormen Spannungen. Sie betont, wie wichtig es ist, kulturelle und religiöse Werte in die westlich geprägte Medizin und Psychotherapie zu integrieren.

\section{Gespräche über Angst verbinden}

Bei einem Workshop mit Fallbesprechungen ist das gegenseitige Verständnis am grössten. Die vorgestellten Patienten sind vorwiegend Überlebende des IranIrak-Krieges und des Erdbebens in Bam mit schweren posttraumatischen Störungen. Ärger, Wut und körperliche Beschwerden wie Nackenschmerzen und vermehrtes Schwitzen sind häufige Symptome. Die Teilnehmer reden langsam, wir verstehen uns. Es ist eigenartig: Gespräche über lustvolle Dinge wie Tanz, Sex und Wein schaffen Distanz - zu verschieden sind die Kulturen, die Ansichten. Gespräche über Angst, Schmerz, Verletzung und Krankheit dagegen bringen uns näher.

Ein Schweizer Kollege flüstert mir zu, dass unsere Schweizer Angst-Patienten die iranischen Veteranen, die um ihre Existenz kämpfen, treffen sollten. Dies würde vermutlich das Koordinatensystem ihrer eigenen Ängste heilsam durcheinanderbringen.

Die internationalen Kollegen haben grosses Interesse am Schweizer System der beruflichen Wiedereingliederung. Eine Teilnehmerin will wissen, wo sich die führenden Forschungsgruppen der Psychotraumatologie befänden. Wir sind uns darüber einig - in Israel. Doch niemand wagt, hier diese Antwort zu geben. Da ist sie wieder, die Angst.

Wolken über Teheran. Ein seltenes Ereignis. Kurzer Regen nach einer langen Trockenperiode. Die Strassen sind ölig. Während der Fahrt vom Restaurant zum Hotel sehen wir drei schwerverletzte Motorradfahrer. Ein iranischer Arzt erzählt mir, dass Strassenunfälle eine wichtige Ursache von Angststörungen, körperlichen Krankheiten und Invalidität sei. Die Zukunft der Verunfallten sei oft unklar, medizinisch wie ökonomisch, was wesentlich zur Verunsicherung der Unfallopfer beitrage.

\section{Das Gesicht der Angst ist kulturell bedingt}

Am Schluss der Veranstaltung sprechen iranische Künstler und Schauspieler über das Thema Angst. Eigentlich eine gute Idee, da kulturelle Faktoren bei der weltweiten Zunahme von Angststörungen bedeutsam sind. Leider verstehen wir kaum etwas, die Übersetzung ist ungenau und setzt gelegentlich aus. Ein Schauspieler, der lange schweigt, sagt am Schluss, er wisse nicht, was seine Rolle bei diesem Kongress sei. Der Anspruch des Kongresses, Westen und Osten, Kultur, Wissenschaft und Politik zusammenzubringen, um das globale Phänomen Angst zu besprechen, ist hoch. Wir sind erst am Anfang.

\section{Psychosoziale Medizin wirkt im kulturel- len Kontext}

Was war eigentlich das Ziel des Kongresses? Die Iraner wollen ihr Gesundheitswesen in Bezug auf Psychiatrie und Psychosomatik ausbauen. Treiber dieses Ausbaus sind unter anderem die Kriegsveteranen, die in der Öffentlichkeit ein hohes Ansehen geniessen. Ferner sind sich die Politiker und religiösen Führer einig, dass das Regime langfristig nur dann Erfolg haben kann, wenn es gelingt, westliche Werte und Individualismus, die von der jungen Generation eingefordert werden, in die religiös geprägte Tradition zu integrieren. Nicht nur die psychosozial orientierte Medizin, auch die Forschung auf diesem Gebiet soll gefördert werden. Dies wird es den Iranern ermöglichen, eigene Interventionen und Therapien zu entwickeln und auszutesten. Das ist wichtig, weil es keine psychosoziale Medizin gibt, die losgelöst vom kulturellen Kontext wirkt.

Was nehme ich aus Teheran mit? Angst ist universell. Die Ursachen mögen verschieden sein, das Gefühl ist das Gleiche. Wir sollten im internationalen Dialog nicht vergessen, über die Themen zu sprechen, die uns näherbringen. Dazu können wir Ärztinnen und Ärzte einen Beitrag leisten. 